\title{
EL FUTURO DE LOS ESPACIOS RURALES COMUNITARIOS TRAS LAAMPLIACIÓN DE LA UE-25 *
}

\author{
Edmundo BAYÓN BUENO \\ Junta de Castilla y León
}

En nuestra Unión Europea recientemente ampliada a 25 Estados miembros, las zonas rurales representan la mayor parte de su territorio y albergan a gran parte de su población, estando caracterizadas por la amplia variedad de matices de las distintas zonas rurales de Europa en cuanto a sus paisajes naturales, sus sistemas de producción agraria, su capacidad de retener y de atraer población, por el papel que juega la agricultura y por la diversificación de la economía local.

Existe una problemática común en la mayoría de las zonas rurales europeas, ya debatida en diversos foros como la Conferencia sobre Desarrollo Rural de Salzburgo en octubre de 2003, destacando principalmente la dificultad de acceso a los servicios públicos, la baja densidad de población, la falta de empleo alternativo y la estructura de edad de la población que reducen considerablemente su potencial de desarrollo, especialmente en lo que se refiere a las oportunidades de las mujeres y los jóvenes.

Con la adhesión de 10 países nuevos, la Unión Europea aumentó el 1 de mayo la población en un $20 \%$, la superficie, en el $23 \%$ y la riqueza solamente en el 4 $\%$, y por el contrario el número de las explotaciones agrícolas se incrementó en el $74 \%$ y el de los agricultores en un 56\%. Es por ello que los nuevos países incorporados, además de la problemática descrita, deberán afrontar la mejora en la competitividad del sector agrario, y es de prever que dará lugar a una fuerte reestructuración agraria por lo que necesitará un apoyo especial de la política de desarrollo rural de la UE a estos nuevos Estados Miembros.

Si hacemos un análisis de lo que tenemos en Castilla y León, la problemática puede ser parecida, aunque con otros niveles de renta. Nuestra región, con $94.204 \mathrm{~km}^{2}$ de superficie, repartidos entre el conjunto de las 9 provincias, con 2.247 municipios y 6.350 núcleos de población, donde unos $90.000 \mathrm{~km}^{2}$ corresponden al medio rural (considerando como tal la superficie ocupada por los

\footnotetext{
* Aportación al XII Coloquio de Geografía Rural ¿Qué futuro para los espacios rurales? León, 15-17 de septiembre, 2004. Mesa redonda El futuro de los espacios rurales comunitarios tras la ampliación.
} 
municipios inferiores a 10.000 habitantes); o dicho de otra manera, en una Comunidad Autónoma, donde el 95\% de la superficie es rural, da una idea clara de la importancia y el peso especifico que tiene para nosotros el medio rural. El $5 \%$ de la superficie restante se reparte entre 20 municipios (entre los que se encuentran las nueve capitales de provincia). Además, en nuestro territorio rural residen 1.039.283 personas, es decir el 41,8\% del total de la Comunidad Autónoma, con lo que la densidad media poblacional del mismo alcanza escasamente los 11,5 habitantes $/ \mathrm{km}^{2}$

A la vista de estos datos, mantener un nivel de vida digno para los que habitan en áreas rurales, es un objetivo fundamental cuya magnitud desborda los límites de la Administración Agraria y donde la ordenación del territorio debe jugar un importante papel en el que se estructuren debidamente los servicios públicos necesarios y adecuados. Prueba de ello, es que se ha creado en las Cortes de Castilla y León una "Comisión sobre la evolución de la población rural en Castilla y León" que está analizando el problema desde todas las perspectivas posibles.

Predecir cual va ser el futuro de los espacios rurales comunitarios a nadie se nos escapa que es una tarea harto complicada. Nuestra experiencia en la aplicación de la política de desarrollo rural en Castilla y León en estos últimos años nos ha permitido reflexionar sobre las debilidades del mundo rural y, si bien todas las áreas tienen, con carácter general, los mismos síntomas -escasa densidad y envejecimiento de la población, etc.-, ¿tienen los mismos remedios? Naturalmente que no. Cada zona tiene recursos e idiosincrasia distintos, debilidades y fortalezas diferentes y se debe afrontar la problemática de manera distinta. Es por ello que la aplicación de los mismos remedios no valen para situaciones geográficas distintas y sí en cambio para aquellas que tienen un elevado grado de homogeneidad. La teoría del "café para todos" surte el mismo efecto que surtiría en un caso sanitario, si a todos los enfermos que entran en un hospital con un síntoma febril, se les aplicara el mismo remedio, sin investigar las causas que provocan tal anomalía. Ese hospital está llamado al fracaso y puede que corramos el mismo riesgo en cuanto a los planes de desarrollo rural, si pretendemos aplicar esta uniformidad para todos.

Por tanto, los Programas de Desarrollo deben abordarse a través de áreas homogéneas, cualquiera que sea su magnitud o incluya espacios territoriales colindantes, dependientes de unidades administrativas diferentes, lo que no debiera impedir, ni mucho menos, que exista una buena interconexión entre ellas.

Dicho esto, conviene sacar la conclusión de que un buen Programa debe ser diseñado por quienes sufren las consecuencias de las carencias de este medio, pues si todos estamos de acuerdo en que nadie conoce mejor un pueblo que 
quienes viven en él, y lo afirmamos axiomáticamente, tal aseveración es perfectamente extensible a un área homogénea. Hay mil ejemplos que podrían ponerse al respecto y mil más de fracasos de quienes intentaron "hacer felices" a los habitantes de una comarca por un procedimiento que no gustaba a ninguno de aquellos a quien iba dirigido.

Por otro lado, un Programa de Desarrollo Rural ha de contemplar todos los aspectos de la vida de un área homogénea, porque todos están interrelacionados y solamente a efectos de estudio, análisis y diseño podemos separarlos.

Es frecuente, sin embargo, que esta separación termine aislándose y se convierta en acción, siendo esta separación responsable, en muchos casos, de no pocos fracasos. Quienes pretendan promocionar, por ejemplo, el turismo rural, no deben estar desconectados de quienes sean responsables de la mejora de las comunicaciones, ni éstos y aquellos de las restantes estructuras productivas (agricultura, artesanía, etnografía, etc.) y todos ellos arropados, protegidos y amparados por un medio ambiente natural conservado y mejorado en su caso.

No olvidemos tampoco que no hay desarrollo si no hay actividad económica y ésta ha de estar sostenida por actividades que formen un tejido, sin el cual el resto de ellas se convierten en medidas de buena voluntad que no proporcionan más desarrollo que el de las buenas intenciones.

Desde este punto de vista, la primera necesidad está en diversificar la economía, a través de actividades no agrarias que colaboren con las que sí lo son: turismo rural, producciones artesanales, pequeñas industrias de transformación, talleres, etc.; en general, creación de pequeñas empresas en las que la mujer debe tener un protagonismo especial.

En este aspecto, tampoco el medio rural es homogéneo y en él habrá que distinguir tres grandes grupos bien diferenciados: las áreas rurales periurbanas que tienen muchas más facilidades para diversificar su economía; las áreas rurales propiamente dichas en las que la diversificación es más difícil y donde habría que promover económica y fiscalmente actividades extra-agrarias o de transformación de los productos en ellas obtenidos y, finalmente lo que pudiéramos llamar las áreas rurales profundas, donde quizá tengan más sentido factores de desarrollo que apoyándose en actividades tradicionales abran nuevos yacimientos de empleo.

Conviene señalar también que se han desarrollado y se siguen desarrollando actuaciones de desarrollo rural directamente relacionadas con las zonas rurales y que han sido incluidas a lo largo del tiempo dentro de las distintas políticas en materia de agricultura. 
Habría que destacar en este sentido los grandes planes y obras llevados a cabo en materia de concentración parcelaria, regadíos y otras infraestructuras, consiguiendo en conjunto un mejor ordenamiento de la propiedad, con la influencia directa que estas actuaciones tienen sobre las mejoras de la calidad de trabajo de los agricultores y el incremento de la productividad, puntos ambos en los que también han tenido vital importancia otros como la industrialización y transformación agroalimentaria, la formación y las mejoras en las técnicas productivas, la modernización y mejora de las explotaciones agrarias, la comercialización de productos y todo, en definitiva, con el fin de alcanzar una mayor rentabilidad de las explotaciones agrarias, sin olvidar todas aquellas cuyo destino principal es el medio rural tanto en su vertiente física como en la poblacional. Tampoco se pueden olvidar las políticas sobre ordenación de bosques y actuaciones sobre medio ambiente, así como programas de investigación y otros de inversiones cofinanciados con fondos europeos o nacionales, bien sean del estado o autonómicos.

Todos estamos concienciados de la necesidad de paliar las debilidades de nuestro mundo rural, aprovechando los recursos y posibilidades que nos ofrece y aplicando medidas complementarias que hagan posible la diversificación de actividades que permitan la creación de nuevos puestos de trabajo, que contribuyan a mejorar la calidad de vida en el medio rural, que sean capaces de mejorar las infraestructuras, servicios, dotaciones, etc., que permitan atraer inversiones empresariales que creen empleos, fijen población y frenen en lo posible el abandono de nuestros pueblos y contribuyan así al mantenimiento de sus valores intrínsecos.

También hemos adquirido experiencia en la aplicación de la política de desarrollo rural mediante el concepto de «bottom-up» o "abajo-arriba", ya que se incide, en las nuevas directrices, en la importancia y la necesidad de que sean los propios habitantes del medio rural, directamente o a través de las instituciones u organizaciones en las que están representados, los que planteen cuáles son sus necesidades principales y las soluciones más convenientes para paliarlas, tratando siempre de aprovechar las potencialidades existentes. Me estoy refiriendo a la Iniciativa LEADER y al programa operativo PRODER gestionados por Grupos de Acción Local. Ciertamente, el procedimiento puede colaborar de forma muy positiva, pero con la salvedad de que el desarrollo propio que se busca no termine convirtiéndose en el desarrollo de quienes lo lideran o lo promueven, debiendo reconocer también que su tarea no es fácil y que en muchas de nuestras comarcas hay autentica dificultad para encontrar promotores capaces de aportar iniciativas y afrontar nuevos proyectos.

Respecto al futuro de las políticas de desarrollo rural, creo que debemos ser optimistas puesto que la Comisión Europea ha realizado propuestas en el senti- 
do de reforzar las políticas del denominado "segundo pilar" de la PAC. Ha planteado la propuesta de un paquete de medidas de desarrollo rural, fijando un crédito global financiero para el periodo 2007-2013 de 88.750 ME que corresponden a unos 13.700 ME anuales. Es por tanto que va a seguir existiendo financiación europea para los Programas de Desarrollo Rural, si bien para Castilla y León, que va a salir de las Regiones Objetivo $n^{\circ} 1$, la cofinanciación europea es presumible que se irá reduciendo a lo largo del periodo de transición con un descenso paulatino de la cofinanciación europea en los proyectos de desarrollo rural.

Por lo que se va conociendo, los programas de la nueva generación serán mejores, más amplios y más sencillos, al menos eso es lo que nos cuentan.

Las principales características establecidas para el futuro en la política de desarrollo rural se van a centrar en:

- Un solo instrumento de financiación y programación, el nuevo Fondo Europeo Agrícola de Desarrollo Rural (FEADER).

- Una genuina estrategia comunitaria de desarrollo rural con un mayor énfasis en las prioridades de la UE.

- El refuerzo del control, la evaluación y la elaboración de informes.

- Un enfoque ascendente («bottom-up») consolidado. Los estados miembros, regiones y grupos de acción local tendrán mayor peso a la hora de ajustar los programas a las necesidades locales.

Los tres objetivos principales de esta nueva política van a ser:

1. Mejorar la competitividad de la agricultura y la silvicultura:

- Mejora y desarrollo de las infraestructuras relacionadas con el desarrollo y la adaptación de la agricultura y la silvicultura.

- Apoyo a los agricultores que participen en los programas de calidad de los alimentos.

- Instalación de jóvenes agricultores.

- Ayuda a los agricultores de «semi-subsistencia» de los nuevos Estados miembros para ser competitivos.

2. Medio ambiente y gestión del suelo

- Ayudas para compensar las desventajas naturales para los agricultores de las Zonas de Montaña. 
- Ayudas de Red Natura 2000

- Medidas agroambientales

- Ayudas en favor del bienestar de los animales

- Las medidas agroambientales seguirán siendo obligatorias.

3. Mejora de la calidad de vida y diversificación

- Diversificación hacia actividades no agrícolas

- Ayuda para la creación de micro-empresas

- Promoción del turismo

- Renovación de núcleos de población rurales

En cuanto a la iniciativa LEADER se pretende dar un nuevo enfoque. Cada programa debe tener un elemento LEADER para la aplicación de las estrategias de desarrollo local de los grupos de acción local. 